\title{
Interstitial brachytherapy for bladder cancer with the aid of laparoscopy*
}

\author{
Annemieke Nap-van Klinken, RTT', Saskia J.E.A. Bus, RTT', Theodorus G. Janssen, RTT', Marion P.R. Van Gellekom, PhD!, \\ Geert Smits, MD, PhD², Elzbieta Van der Steen-Banasik, MD' \\ 'Department of Radiation Oncology, Arnhem Radiotherapy Institution (ARTI), Arnhem, 2Urology Department, Rijnstate Hospital Arnhem. \\ Anhem, Netherlands
}

\begin{abstract}
Since 2009, 40 patients with a T1/T2 bladder cancer have been treated at ARTI with interstitial brachytherapy via laparoscopy. Under general anaesthesia, the tumour area is implanted under cystoscopic control with the aid of a laparoscope, and instruments are attached to the Da Vinci robot. Mapping is then done via a simulator photo and a CT scan. With this method, the patient has fewer complications and the average hospitalization time is halved, while a consistent quality of implant is maintained. This method is also associated with a major reduction in the number of problems that can be attributed to the accessibility of the catheter.

J Contemp Brachytherapy 2014; 6, 3: 313-317 DOI: $10.5114 / j \mathrm{jcb} .2014 .45492$

Key words: bladder cancer, brachytherapy, interstitial, laparoscopy.

*This article is a translation from the Dutch article "Interstitiële brachytherapie bij het blaascarcinoom met behulp van laparoscopie" published in GAMMA professional, January 2014, volume 64, number 1, pages 17-21 - the magazine of the Dutch Society of Medical Imaging and Radiotherapy. Translation was done with permission of GAMMA professional.
\end{abstract}

\section{Purpose}

Patients with bladder cancer have been treated with interstitial brachytherapy at the Arnhem Radiotherapy Institution (ARTI) since 1973. Initially this treatment was carried out with radium needles followed by cesium needles. The institute switched to implanting catheters in 1996. Until June 2009, catheters were implanted via an open procedure. Nowadays, the catheters are placed via laparoscopy and, since September 2010 with the aid of the Da Vinci robot (Da Vinci ${ }^{\circledR}$ Surgical System, Intuitive Surgical, Inc., Sunnyvale, CA, USA). This has advantages in many areas.

\section{Context}

In the Netherlands approximately 5,200 people are diagnosed with bladder cancer every year. The standard treatment of invasive bladder tumours is cystectomy. For patients with a solitary T1G3-T2 urothelial cancer less than $5 \mathrm{~cm}$, treatment to save the bladder by using brachytherapy combined with external radiation is considered. Earlier studies show that brachytherapy results in a local control of between $70 \%$ and $88 \%$. The five-year survival rate varies between $48 \%$ and $73 \%$ [1-3]. Approximately $90 \%$ of patients cured by radiotherapy retain an effectively functioning bladder $[4,5]$.
Between 1996 and 1998, patients with bladder cancer were irradiated at ARTI with a manual afterloading method using a low-dose-rate (LDR) iridium wire. Since the introduction of the pulse dose rate (PDR) afterloader $(\mathrm{Nu}-$ cletron, an Elekta company, Elekta AB, Stockholm, Sweden) in 1998, 77 patients at ARTI have been irradiated by means of interstitial brachytherapy. Forty patients have been treated via laparoscopy, 32 of whom were treated with the aid of the Da Vinci robot. Patients with stage T2 cancer received 20 fractions of 2 Gy externally, followed by a brachytherapy boost of 25 Gy in 10 pulses (33 patients via laparoscopy) or $28 \mathrm{~Gy}$ in 28 pulses (26 patients via an open procedure and 6 patients via laparoscopy). All regimens were administered within office hours only. Twelve patients followed a different radiation regimen.

\section{Implantation of the catheters}

The use of laparoscopy for implanting the catheters has made the operation less invasive. The patient is given a general anaesthetic and is placed in the Trendelenburg position. The laparoscope and instruments are inserted into the abdominal cavity through small incisions in the skin, and subsequently connected to the Da Vinci robot. The tumour area in the bladder is mapped with the aid
Address for correspondence: Annemieke Nap-van Klinken, RTT, Department of Radiation Oncology, Arnhem Radiotherapy Institution (ARTI), Arnhem, Netherlands, Wagnerlaan 47, 6800JD Arnhem, Netherlands, phone: +31 263712412, e-mail: a.nap@arnhemrti.nl
Received: 05.09.2014

Accepted: 06.09.2014

Published: 30.09 .2014 


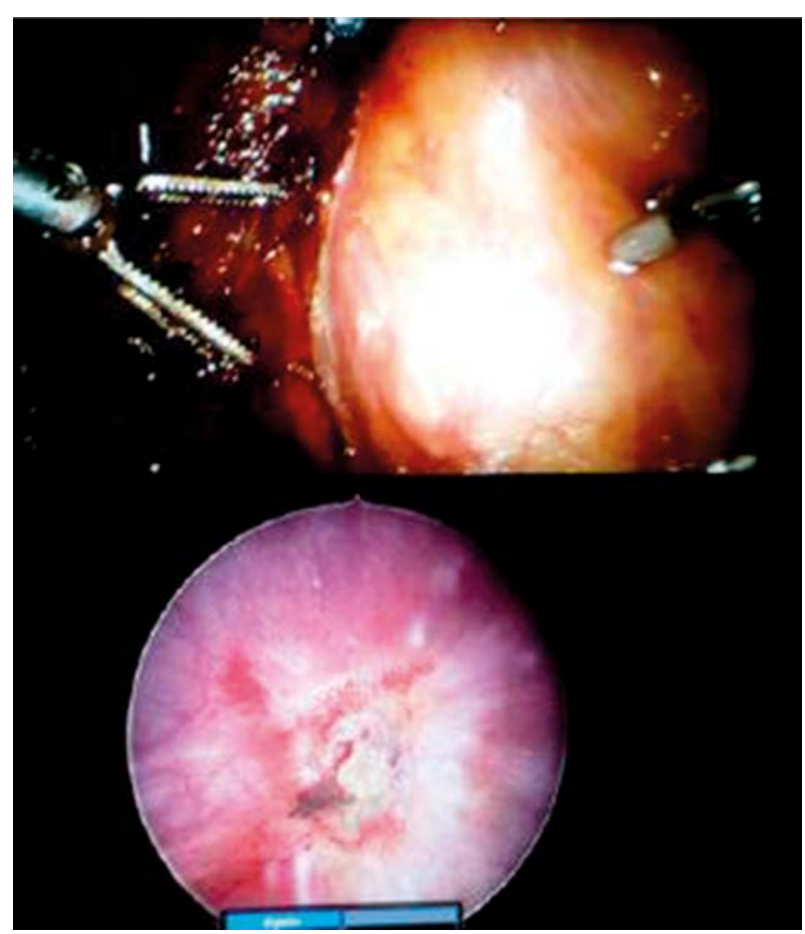

Fig. 1. The illustration at the bottom shows the tumour area in the bladder via cystoscopy. In the illustration at the top, the cystoscope light is visible in the abdominal cavity via the laparoscope

of a cystoscope. The light of the cystoscope is visible in the abdominal cavity via the laparoscope (Fig. 1). This is the area where the catheters are implanted. In some instances a partial cystectomy is performed before the catheters are implanted.

The catheters can be implanted once the surrounding structures have been moved away from the implantation area. The radiotherapist inserts the needle with the attached catheter through the patient's skin, after which it is handled by the robot operated by the urologist. With the aid of the robot, the catheter is guided further into the abdominal cavity and through the bladder wall under cystoscopic control. The needle with the catheter is then pushed towards the outside through the skin on the other side of the abdomen. The needle is then removed from the catheter.

All the catheters are inserted in this way (Fig. 2). Clips are placed on both sides of the clinical target volume (CTV). These are visible on the CT scan and the X-rays. The catheters are fixed on both sides of the abdomen with buttons. The incisions in the skin are then sutured and the patient is transferred to the recovery room.

The catheters are prepared during the laparoscopy. The inner catheter is pushed down as far as possible; this stiffens the catheter and protects it against kinking $0.5 \mathrm{~cm}$ of the catheter is cut off. A slightly curved $4.5 \mathrm{~cm}$ needle (Nucletron, an Elekta company, Elekta AB, Stockholm, Sweden), specifically developed for this procedure, is attached to the catheter. Small ridges on the needle ensure that it is firmly fixed in the catheter. The needles are only used once because, during implantation, the area where

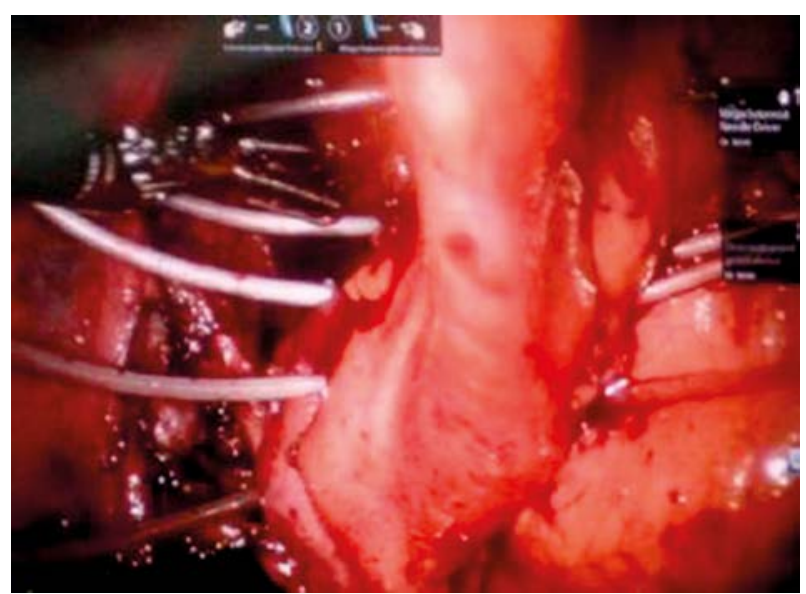

Fig. 2. Laparoscopy image of 5 catheters implanted

in the bladder wall parallel to each other

the needle is connected to the catheter is tightly squeezed, which means that the needle may become detached from the catheter during the next implantation.

\section{Mapping/planning}

Mapping for the purpose of the radiation plan is done using an anterior-posterior X-ray and a CT scan. The catheter is shortened on the simulator on the basis of the $\mathrm{X}$-ray from the open side (the side where the needle was attached). In order to determine the length of the catheter, a thin metal wire of fixed length is introduced into the catheter. The end of the metal wire indicates the first source position. This must be located just past all the clips. This process is repeated for all the catheters (Fig. 3). Also, a CT scan is made with the metal wires inserted in the catheters. The slice thickness used is $1 \mathrm{~mm}$, and the CT slices are imported into the Flexiplan (Nucletron, an Elekta company, Elekta AB, Stockholm, Sweden) planning program. The catheters are reconstructed on the CT slices and a step size of $2 \mathrm{~mm}$ between the source positions is selected. The active dwell positions lie between the clips, which are left behind during the implantation. The dose points are located $5 \mathrm{~mm}$ from the catheters. The dwell times are calculated by dosing to an average of all the dosing points and sometimes improvements are made manually. However, increasing the dwell times should be done with caution in order to avoid too high a dose around the catheters (Fig. 4).

The first pulse is often given on the day that the catheters are implanted. After the last pulse, the catheters are removed from the patient. The catheter is cut under the button and then pulled out of the abdomen on the other side.

\section{Acute and late complications}

Compared with the open procedure, implantation via laparoscopy has both physical and logistic advantages for the patient. The operation, as performed at present, is minimally invasive, resulting in practically no blood loss and a shorter operating time. The reduction in hospitalization time, from an average of 16 days (6-90 days) to 


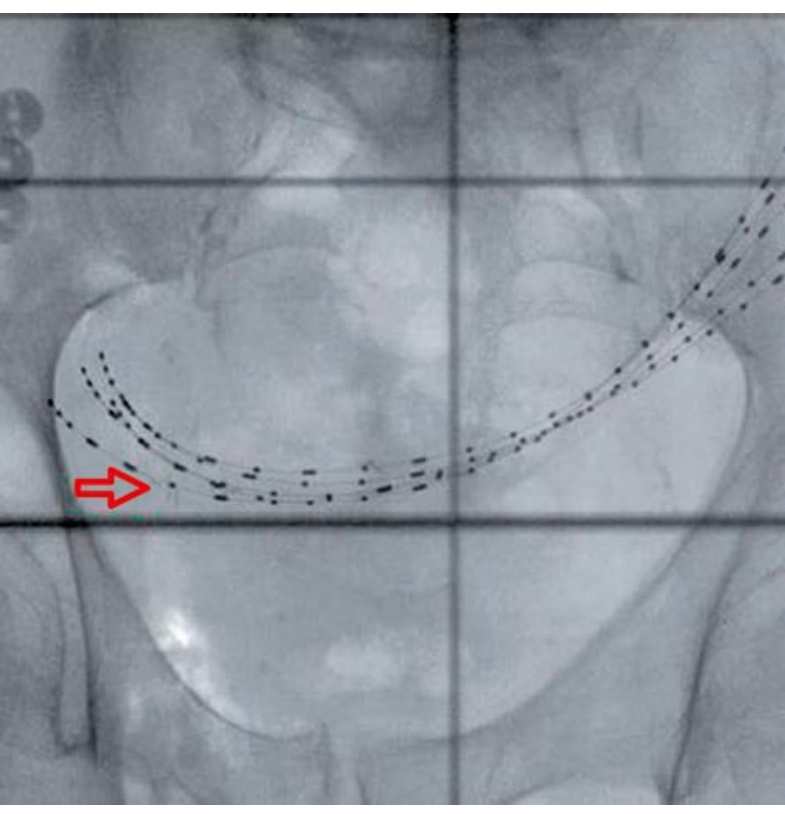

Fig. 3. X-ray anteroposterior photo of a patient where 5 catheters are implanted in the bladder wall. The catheters are visible because of the metal wires. The clips are also visible in the photo; 2 of the 8 clips can be seen near the arrow

an average of 6.5 days (4-18 days) is particularly striking. Wound healing after an open procedure takes longer; one of the patients developed a serious bladder defect, which led to 90 days hospitalization. One patient developed aspiration pneumonia and 1 patient had urine leakage after the partial cystectomy of a recurrent urachal tumor. After a period with an indwelling catheter, the defect underwent secondary closure. Up until now, late complications have consisted of asymptomatic ulcers and one abscess of the TUR cavity in one patient, one year after TURB for in situ carcinoma. This patient was treated with antibiotics and hyperbaric oxygen.

\section{Quality of the implant}

One of the important aspects, which contributes to the success of interstitial brachytherapy of the bladder is the quality of the implant. The catheters have to lie in parallel with approximately $1 \mathrm{~cm}$ distance from each other in order to achieve a dose distribution, which is as homogeneous as possible [6]. The quality of the implant is assessed on the basis of the following implant-specific parameters: homogeneity index (HI) and overdose index (OI). Since the CTV and the organs at risk were not entered, the targetspecific parameters could not be assessed. The HI indicates the size of the part of the target volume that receives $100 \%$ to $150 \%$ of the prescribed dose (Formula 1) $[7,8]$.

The OI indicates the size of the part of the target volume that receives more than $200 \%$ of the prescribed dose (Formula 2) [7].

Formula 1: $\mathrm{HI}=\left(\mathrm{V}_{100}-\mathrm{V}_{150}\right) / \mathrm{V}_{100} \times 100 \%$

Formula 2: $\mathrm{OI}=\mathrm{V}_{200} / \mathrm{V}_{100} \times 100 \%$

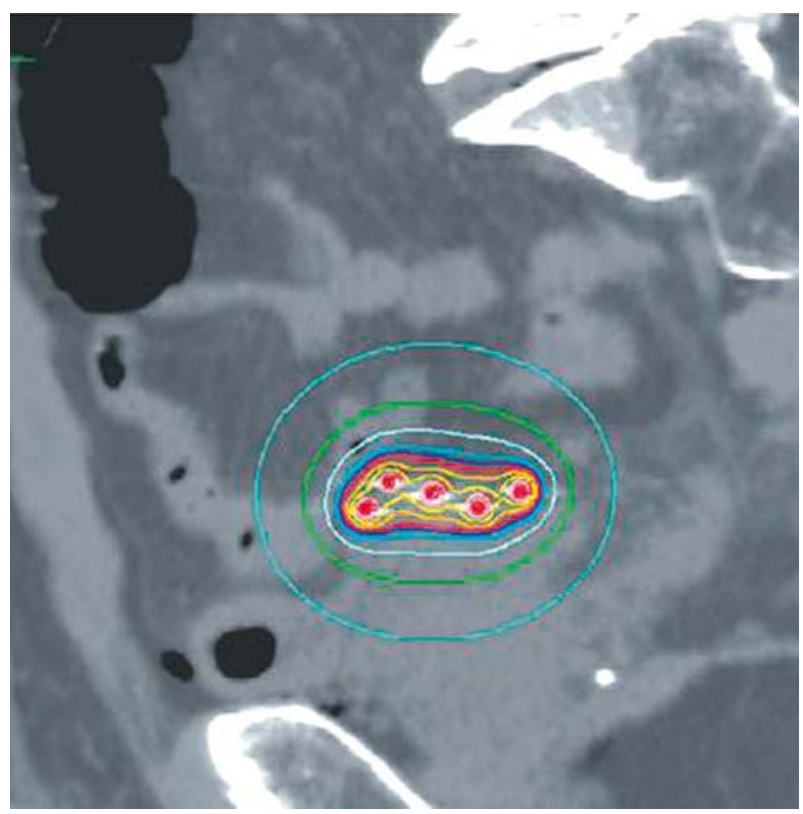

Fig. 4. A sagittal cross-section of a patient where 5 catheters are implanted in the bladder wall. This mapping shows the dose distribution. The red line is the $100 \%$ isodose line

Ten patients were chosen at random from both group 1, who were patients implanted with an open procedure, and group 2, who were patients implanted via laparoscopy. The total active length of the catheters was comparable in both groups. The average total active length of group 1 was $14.75 \mathrm{~cm}$ and group 2, $13.87 \mathrm{~cm}$. Homogeneity index and OI values of both groups are given in Figures 5 and 6 . The average $\mathrm{HI}$ and OI values of group 1 were $57.3 \%$ and $19.8 \%$, respectively. The average $\mathrm{HI}$ and OI values of group 2 were $57.0 \%$ and $21.3 \%$, respectively.

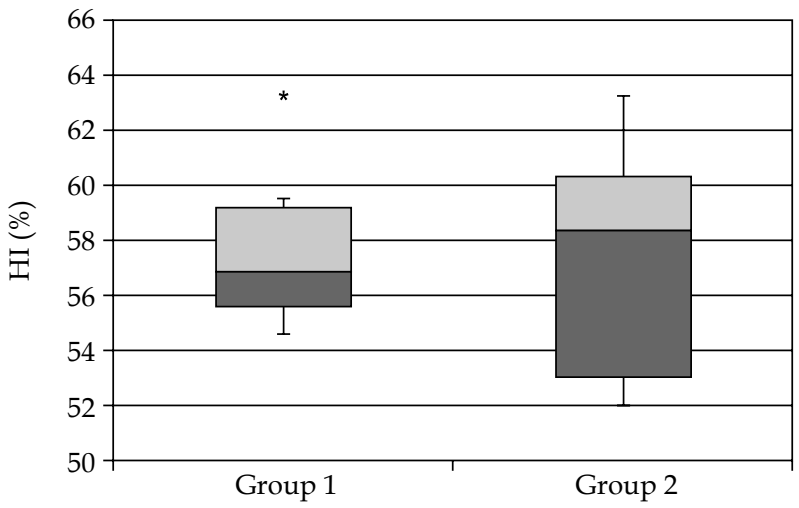

Fig. 5. The homogeneity index (HI) values are indicated in a box plot for both groups. The box contains $50 \%$ of the data ( $25 \%$ percentile up to $75 \%$ percentile). The median is indicated by the horizontal line in the box. The terror bars indicate the minimum and maximum values. If a data point deviates from the box length more than once, it is an extreme point indicated by a *; 1 extreme point occurs in group 1 only 


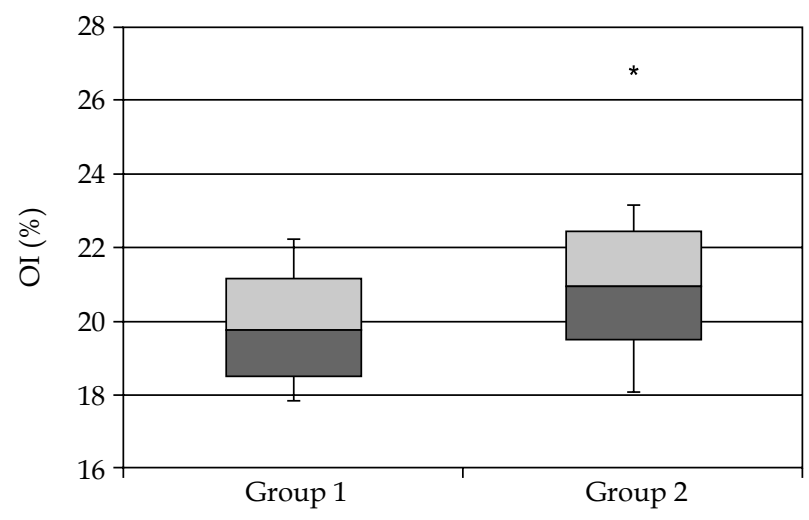

Fig. 6. The overdose index (OI) values are indicated in a box plot for both groups. The box contains $50 \%$ of the data ( $25 \%$ percentile up to $75 \%$ percentile). The median is indicated by the horizontal line in the box. The error bars indicate the minimum and maximum values. If a data point deviates from the box length more than once, it is an extreme point, indicated by a *; 1 extreme point occurs in group 2 only

An unpaired $t$ test $(\alpha<0.05)$ showed whether the parameters of both groups differed significantly. The $p$ values found were 0.16 and 0.85 for $\mathrm{HI}$ and OI, respectively. Since these were bigger than the significance level, no significant difference was shown in the quality of the implants. A trend over time was found in group 2 where the homogeneity of the dose distribution increases, whereas overdosing decreases.

\section{Discussion}

Since 2009, interstitial catheter implantation has been done using a laparoscopic method. In view of the short length of time and the relatively small numbers of patients, no conclusions can be drawn about the clinical results in the long term.

One noticeable result of implantation via laparoscopy is the significant reduction in problems during radiation. In 16 out of 26 patients who had an open procedure, problems were identified with 1 to 6 pulses, which were related to the accessibility of the catheter. Such problems can have consequences for the treatment. If a catheter was no longer accessible for the Ir-192 source, the plan had to be adjusted. In the new plan, the catheter or part of it, is then no longer used. This does not by definition mean that the plan becomes worse. Often, by means of optimizing again, a clinically acceptable radiation plan can still be achieved. For this reason, a new plan was made for 2 of the 26 patients who had received 28 pulses. Since we started using the laparoscopic method, no further problems connected with the accessibility of the catheter have occurred. The reduction in problems since implantation via laparoscopy can be explained by a combination of different factors. The radiation regimen for the treatment is adjusted from 28 pulses to 10, because of the logistic burden for the nursing department. In the case of 10 pulses, the inner catheter was reinserted into the catheter after each pulse, whereas in the case of 28 pulses, the patient remained connected to the afterloader. The later scenario increased the risk of deformation of the catheters due to reduced rigidity.

Also, when implanting via an open procedure, spacers were used, which were placed in the bladder in order to achieve an equal distance between the catheters. The use of spacers guaranteed the quality of the implant. However, the spacer appeared to have an important influence on the deformation of the catheter and subsequently cause an obstruction for the Ir-192 source.

Since our hospital started using the laparoscopic method, the use of spacers has been omitted. In fact it is no longer possible to easily remove the spacers after radiation therapy. As a result of this, the analysis of the quality of the implant is an important step in the evaluation of implantation via laparoscopy. It is not necessary to use Da Vinci robot for implantation of the catheters. However, the robot does make the implantation process easier, because movements can be made with greater precision.

\section{Developments}

In addition to implantation via laparoscopy, our hospital has explored with Elekta Brachytherapy ways to improve the existing catheter. A new catheter has been developed (Luneray ${ }^{\mathrm{TM}}$, Nucletron, an Elekta company, Elekta AB, Stockholm, Sweden) where a needle is already attached. The catheter is supplied pre-assembled as a disposable and is ready for immediate use. At the moment, we are investigating whether the patient's bladder fill influences the quality of the implant. During implantation, the patient's bladder is approximately filled with $150 \mathrm{cc}$. The CT scan and radiation therapy are performed with an empty bladder. Currently, a second CT scan is done with the bladder being filled with $150 \mathrm{cc}$ normal saline via the urinary catheter. We are investigating whether this results in an improvement in the configuration of the catheters and the associated dose distribution.

\section{Conclusions}

Bladder implantation via laparoscopy seems to be an effective technique where the quality of the implant remains guaranteed. The hospitalization time for the patient is halved. The catheter implantation is minimally invasive and the acute complication rate is low. This method also results in a major reduction in the number of problems that can be attributed to the accessibility of the catheter. Of course, good multi-disciplinary cooperation is required between urologists, radiotherapists and support personnel in order to achieve optimal treatment.

\section{Disclosure}

The authors report no conflict of interest.

\section{References}

1. Blank LE, Koedooder K, van Os R et al. Results of bladderconserving treatment, consisting of brachytherapy combined with limited surgery and external beam radiotherapy, for patients with solitary T1-T3 bladder tumours less than $5 \mathrm{~cm}$ in diameter. Int J Radiat Oncol Biol Phys 2007; 69: 454-458. 
2. Koning CC, Blank LE, Koedooder C et al. Brachytherapy after external beam radiotherapy and limited surgery preserves bladders for patients with solitary pT1-pT3 bladder tumors. Ann Oncol 2012; 23: 2948-2953.

3. Van der Steen-Banasik EM, Visser AG, Reinders JG et al. Saving bladders with brachytherapy: implantation technique and results. Int J Radiat Oncol Biol Phys 2002; 3: 622-629.

4. Nieuwenhuijzen JA, Pos F, Moonen LMF et al. Survival after bladder-preservation with brachytherapy versus radical cystectomy. A single institution experience. Eur Urol 2005; 48: 239-245.

5. Dutch national workgroup for bladder cancer guidelines (2011); available at: http://www.oncoline.nl/urotheelcarcinoom-vande-blaas. Last accessed July 2014.

6. Veen RE. Applicators in interstitial radiotherapy, "the Deventer system". Trial report 1994; 83-101.

7. Mould RF, Battermann JJ, Martinez AA et al. Brachytherapy from radium to optimization. Nucletron International B.V. 1994; 303-305.

8. Saw CB, Suntharalingam N. Quantitative assessment of interstitial implants. Int J Radiat Oncol Biol Phys 1991; 20: 135-139. 